\title{
Sobrecarga em trabalhadores de saúde de um complexo hospitalar psiquiátrico no Nordeste brasileiro
}

Overload in health workers in a psychiatric hospital complex in the northeast of Brazil Sobrecarga en trabajadores de salud en un complejo hospitalario psiquiátrico en el noreste de Brasil

Francilene Figueirêdo da Silva Pascoal ${ }^{1,2}$ (B) Solange Fátima Geraldo da $\operatorname{Costa}^{1}$ (c) Patrícia Serpa de Souza Batista ${ }^{1}$ (I) Mariana Albernaz Pinheiro de Carvalho ${ }^{1,2}$ (1) Alana Vieira Lordão ${ }^{1}$ (1) Jaqueline Brito Vidal Batista ${ }^{1}$ (1)

1. Universidade Federal da Paraíba, Programa de Pós-Graduação em Enfermagem. João Pessoa, PB, Brasil.

2. Universidade Federal de Campina Grande. Cuité, PB, Brasil.
Autor correspondente:

Alana Vieira Lordão.

Email: alanavieirap@gmail.com.

Recebido em 12/02/2021.

Aprovado em 02/06/2021.

DOI:https://doi.org/10.1590/2177-9465-EAN-2021-0018

\section{Resumo}

Objetivo: este artigo visa identificar os aspectos que causam a sobrecarga laboral em um complexo psiquiátrico e verificar estratégias de minimização desta sobrecarga. Método: os dados foram coletados com trabalhadores de um complexo hospitalar psiquiátrico na cidade de João Pessoa, Paraíba, Brasil. Aplicou-se a Escala de Avaliação do Impacto do Trabalho em Serviços de Saúde Mental (IMPACTO-BR) na coleta de dados, os quais foram processados no software Interface de $R$ pour les Analyses Multidimensionnelles de Textes et de Questionnaires (IRAMUTEQ) e submetidos à análise de conteúdo. Resultados: os resultados foram sistematizados em duas categorias temáticas: Aspectos de sobrecarga laboral e Estratégias para amenizar a sobrecarga. Foi relevado que as maiores causas de sobrecarga são do contexto organizacional, enquanto que o menor impacto advém do paciente. Conclusão e implicações para a prática: as estratégias sugeridas discorreram desde a aquisição de um trabalho digno, com direitos trabalhista, até as ações voltadas para um melhor atendimento para o paciente. Sugere-se que sejam implementadas ações políticas e institucionais de incentivo à integração da equipe, valorização do trabalho e cuidado da saúde física e mental do trabalhador.

Palavras-chave: Condições de Trabalho; Equipe de Assistência ao Paciente; Saúde do Trabalhador; Esgotamento Profissional; Hospitais Psiquiátricos.

\section{Abstract}

Objective: this article aims to identify the aspects that cause work overload in a psychiatric hospital and to list strategies to reduce the overload reported by the 188 participants. Method: data were collected from workers at a psychiatric hospital in the city of João Pessoa, Paraíba, Brazil. A scale for assessing the impact of work in mental health services (IMPACTO-BR) was used, processed in the IRAMUTEQ software and submitted to content analysis. Results: the results were systematized in two thematic categories: Work overload aspects and Strategies to reduce overload. It was revealed that the biggest causes of overload are from the organizational context, while the smallest impact comes from patients. Conclusion and implications for the practice: the suggested strategies ranged from acquiring decent work, with labor rights, to actions aimed at better patient care. It is suggested that political and institutional actions be implemented to encourage team integration, valorization of work and physical and mental health care of workers.

Keywords: Working Conditions; Patient Care Team; Occupational Health; Professional Burnout; Psychiatric Hospitals.

\section{REsumen}

Objetivo: este artículo pretende identificar los aspectos que causan la sobrecarga laboral en un complejo psiquiátrico y las estrategias para minimizar la sobrecarga referida por los 188 participantes. Metodo: los datos fueron recolectados de trabajadores de un complejo hospitalario psiquiátrico en la ciudad de João Pessoa, Paraíba, Brasil. Se utilizó una escala para evaluar el impacto del trabajo en los servicios de salud mental en la recopilación de datos, que fueron procesados en el software IRAMUTEQ y sometidos al análisis de contenido. Resultados: los resultados fueron sistematizados en dos categorías temáticas: Aspectos de sobrecarga de trabajo y Estrategias para amenizar la sobrecarga. Se ha relevado que las mayores causas de sobrecarga son del contexto organizacional, mientras que el menor impacto viene del paciente. Conclusión e implicaciones para la práctica: las estrategias sugeridas discurrieron entre la adquisición de un trabajo digno, con derechos laborales, a las acciones dirigidas a una mejor atención al paciente. Se sugiere que se implementen acciones políticas e institucionales de incentivo a la integración del equipo, valorización del trabajo, y de cuidado de la salud física y mental del trabajador.

Palabras-clave: Condiciones de Trabajo; Grupo de Atención al Paciente; Salud Laboral; Agotamiento Profesional; Hospitales Psiquiátricos. 


\section{INTRODUÇÃO}

O serviço hospitalar psiquiátrico é um recurso da área da saúde ainda muito utilizado na atualidade para tratamentos de pessoas com transtorno mentais. É tido como um local de restabelecimento da saúde, de acesso à alimentação, aos medicamentos e às atividades de higiene. Contudo, também é visto como um ambiente inerte, hostil, que reproduz um cuidado desumanizado ${ }^{1-3}$.

O trabalhador, responsável pelo cuidado no serviço de saúde mental, muitas vezes trabalha sob condições insalubres, escassez de recursos e a ameaça de ser agredido por algum paciente. Tais condições podem gerar uma carga laboral acima da capacidade pessoal e/ou profissional, e, consequentemente, impactar negativamente na sua qualidade de vida e na sua saúde ${ }^{4}$.

Segundo Dejours ${ }^{5}$, as condições de trabalho incluem: ambiente físico, químico e biológico, condições de higiene, de segurança e ergonômicas. Já as questões relacionadas à organização consistem em: divisão e conteúdo do trabalho, sistema hierárquico, modalidades de comando e controle, relações de poder e modo operatório.

Estudos têm demonstrado que más condições laborais e organização ineficiente em serviços psiquiátricos, sobrecarrega os trabalhadores, predispõem os trabalhadores ao desenvolvimento de patologias, como fadiga por compaixão, depressão, estresse e burnout. São patologias que provocam implicações negativas na qualidade de vida do trabalhador, diminuição da eficiência do trabalho e altas taxas de rotatividade de pessoal ${ }^{6-8}$.

Assim, identificar a sobrecarga e intervir nos aspectos causais é relevante para promoção da saúde dos trabalhadores e para a qualidade do atendimento ofertado aos usuários e à Instituição. Nesse sentido, é inegável a importância de novas pesquisas que busquem abordar sobre o impacto no trabalho nos serviços psiquiátricos, tendo em vista que é no contexto laboral que se revelam os maiores fatores de sofrimento e de adoecimento do trabalhador ${ }^{9,10}$.

A partir da investigação bibliográfica (Scielo, LILACS, PubMed, Scopus e BDENF), considerando o recorte temporal das publicações dos últimos 5 anos anteriores à coleta, foi identificada uma escassez de estudo que avaliassem o impacto do trabalho em um complexo psiquiátrico, realizada com a equipe de assistência ao paciente multiprofissional composta por: assistentes sociais, bibliotecário, cirurgião-dentista, educador físico, enfermeiro, farmacêutico, fisioterapeuta, médico, nutricionista, psicólogo, cuidador, trabalhador do apoio/vigilância, auxiliar e técnico de enfermagem, auxiliar de odontologia, oficineiro e terapeuta holística. Foram identificados estudos que avaliassem categorias profissionais especificas ${ }^{11}$ ou realizadas com uma equipe multiprofissional ${ }^{12}$, mas não foram encontrados estudos que incluíssem uma variedade tão ampla de trabalhadores, tampouco que abordassem a temática, conforme proposto no estudo em tela.

Diante do exposto, este estudo tem por objetivo identificar os aspectos que causam a sobrecarga laboral em um complexo psiquiátrico e verificar estratégias de minimização desta sobrecarga.

\section{MÉTODO}

Trata-se de um estudo descritivo, exploratório, de abordagem quanti-qualitativa, realizado em um complexo psiquiátrico $(\mathrm{CP})$ no nordeste brasileiro. Dos 320 trabalhadores pertencentes à equipe multiprofissional que prestam assistência na instituição estudada, 188 participaram deste estudo, sendo 44 técnicos e auxiliares de enfermagem, 38 cuidadores, 33 trabalhadores do apoio/vigilância, 27 enfermeiros, 17 assistentes sociais, 10 psicólogos, 5 farmacêuticos, 3 nutricionistas, 2 cirurgiãs-dentistas, 2 educadores físicos, 1 auxiliar de odontologia, 1 oficineiro, 1 terapeuta holística, 1 bibliotecário, 1 fisioterapeuta, 1 médico clínico, 1 médico psiquiatra.

Para a seleção da amostra, foram adotados os seguintes critérios de inclusão: trabalhadores que possuíam idade igual ou superior a 18 anos e que atuavam, no mínimo, 6 meses na Instituição selecionada para a pesquisa proposta, período de desenvolvimento do burnout. Foram excluídos trabalhadores que apresentaram presença de transtornos mentais e/ou qualquer doença severa que impediam a coleta de dados, que estavam de férias ou de licenças médicas, assim como profissionais que atuavam na área administrativa, tendo em vista que o burnout é desenvolvido em trabalhadores que mantêm contato direto com outras pessoas.

A coleta de dados ocorreu em um CP localizado na cidade de João Pessoa, Paraíba, Brasil. Os participantes receberam um envelope contendo os seguintes instrumentos autoaplicáveis: 1. Questionário com variáveis sociodemográficas, ocupacionais, hábitos de vida e saúde; 2 . Escala de Avaliação do Impacto do Trabalho em Serviços de Saúde Mental (IMPACTO-BR). Posteriormente, os envelopes foram recolhidos pelos pesquisadores.

A Escala é composta por 18 questões quantitativas e 03 questões descritivas complementares. Para atender o objetivo proposto neste estudo, foram utilizadas apenas as questões descritivas: quais são os aspectos do seu trabalho neste serviço que resultam em mais sobrecarga para você? Quais são os aspectos do seu trabalho neste serviço que resultam em menos sobrecarga para você? Quais são os aspectos do seu trabalho neste serviço que você gostaria de mudar para diminuir a sobrecarga? ${ }^{13}$

As respostas referentes às questões descritivas existentes na IMPACTO-BR foram reunidas em um único arquivo, denominado corpus. Para processar os dados existentes no corpus, utilizouse o software IRAMUTEQ (Interface de $R$ pour les Analyses Multidimensionnelles de Textes et de Questionnaires) 0.7 alpha 2, que tem por finalidade encontrar a informação essencial contida em um texto por meio da análise estatística textual, do tipo: estatísticas lexicais clássicas; pesquisa de especificidades de grupos; Classificação Hierárquica Descendente (CHD); análises de similitude e nuvem de palavras ${ }^{14,15}$.

Neste estudo, utilizou-se a análise de similitude e CHD. A análise de similitude organiza graficamente as palavras, identificando a coocorrência e conexidade entre termos, auxiliando na compreensão da estrutura da representação social sobre o objeto em estudo. Com a CHD, o IRAMUTEQ agrupa os 
segmentos dos textos e vocabulários, correlacionando-os por conteúdo em um esquema hierárquico de classes, representado por um dendrograma, que indica a quantidade e composição léxica de classes, a frequência absoluta dos termos e o valor de qui-quadrado agregado ${ }^{14,16}$.

Após o processamento do conteúdo textual, construiu-se o modelo analítico composto por categorias que corresponderam às classes de palavras geradas pela técnica de CHD. Para a análise interpretativa do corpus, utilizou-se a análise de conteúdo, que proporciona uma avaliação profunda e cautelosa dos achados ${ }^{17}$

O projeto foi aprovado pelo Comitê de Ética da Universidade Federal da Paraíba, sob Parecer $\mathrm{n}$ ㅇ. 2.303.520, em conformidade com a Resolução nำ466/2012 do Conselho Nacional de Saúde, que normatiza as diretrizes éticas para o desenvolvimento de pesquisa envolvendo seres humanos no cenário nacional. Todos os participantes, que consentiram a participação no estudo, assinaram o Termo de Consentimento Livre e Esclarecido. Para garantir o anonimato dos participantes, procedeu-se à identificação aleatória, utilizando a letra $P$ de participante, seguido por numeração ascendente até completar o número total de trabalhadores participantes da pesquisa.

\section{RESULTADOS}

Dentre os participantes $(n=188)$, a maioria era do sexo feminino $(67 \%, n=126)$, possuía ensino básico $(60 \%, n=112)$, com média de idade de 47,8 anos (DP =11,07), vínculo empregatício precarizado $(63 \%, n=120)$, jornada de trabalho superior a 40 horas semanais $(53 \%, n=112)$, possuía outro emprego $(63 \%$, $n=70)$, tempo de profissão inferior aos 10 anos $(51 \%, n=95)$, não praticavam esporte $(62 \%, n=116)$, e não haviam tirado férias nos últimos 2 anos $(77 \%, n=144)$. Referente ao tempo livre, $14 \%$ $(\mathrm{n}=26)$ mencionaram que aproveitavam para dormir, assistir televisão ou fazer trabalhos domésticos.

Com relação ao afastamento do trabalho, dos 188 participantes, $10 \%(n=19)$ declararam ter faltado no último ano, e 13\% $(n=24)$, ter tirado licença médica. Os afastamentos foram provenientes de problemas organizacionais do tipo, atraso salarial, por licença maternidade ou adoecimento, com um caso de diagnóstico de estresse.

Quanto à análise do corpus textual, foram encontradas 2.713 ocorrências de palavras, sendo 531 na forma ativa, com frequência média de 3 palavras para cada forma. Esse corpus foi dividido em 77 segmentos de texto; 56 equivalem a $72,73 \%$ do total de palavras foram equiparadas por meio da $\mathrm{CHD}$, aos quais geraram cinco classes, representadas no dendrograma, exposto na Figura 1. Considera-se uma análise adequada dos dados coletados quando há uma retenção de segmentos de texto superior a $70 \%{ }^{15}$.

As cinco classes, criadas com base na ocorrência dos termos, originaram dois subcorpora: O primeiro, é formado pelas Classes 3 e 2 , que se referem às estratégias voltadas para reduzir a sobrecarga no trabalho. $O$ segundo, representado pela Classe 1, relaciona-se aos eventos causadores de sobrecarga Esse último subcorpus foi subdividido, originando as Classes 4 e 5, que reforçam os relatados dos participantes aos eventos causadores de maior e menor sobrecarga (Figura 1).

Baseado nos resultados apresentados no dendrograma, foram estabelecidas duas categorias para análise interpretativa do corpus: A primeira, denominada de Aspectos da sobrecarga laboral, representa as respostas das questões 1 e 2, da IMPACTOBR; e a segunda categoria, Estratégias/recomendações para amenizar a sobrecarga, responde à questão 3.

\begin{tabular}{|c|c|c|c|c|c|c|c|c|c|c|c|c|c|c|}
\hline \multirow{2}{*}{\multicolumn{3}{|c|}{$\begin{array}{c}\text { Classe } 3(19,6 \%) \\
\text { Estratégias para a redução da } \\
\text { sobrecarga }\end{array}$}} & \multirow{2}{*}{\multicolumn{3}{|c|}{$\begin{array}{c}\text { Classe } 2(17,9 \%) \\
\begin{array}{c}\text { Estratégias para a redução da } \\
\text { sobrecarga }\end{array} \\
\end{array}$}} & \multicolumn{3}{|c|}{$\begin{array}{c}\text { Classe } 1(21,4 \%) \\
\text { Sobrecarga }\end{array}$} & \multicolumn{3}{|c|}{$\begin{array}{c}\text { Classe } 4(25,0 \%) \\
\text { Maior sobrecarga }\end{array}$} & \multicolumn{3}{|c|}{$\begin{array}{c}\text { Classe } 3(16,1 \%) \\
\text { Menor sobrecarga }\end{array}$} \\
\hline & & & & & & Palavra & f & $\mathbf{X}^{2}$ & Palavra & f & $\mathbf{X}^{2}$ & Palavra & f & $\mathbf{X}^{2}$ \\
\hline Palavra & f & $\mathbf{X}^{2}$ & Palavra & f & $\mathbf{X}^{2}$ & Alta médica & 100.00 & 15.79 & Falta & 66.67 & 14.14 & Conversar & 83.33 & 18.12 \\
\hline Aumentar & 75.00 & 18.12 & Aumentar & 100.00 & 30.91 & Família & 71.43 & 11.88 & Quando & 45.83 & 11.88 & Problema & 80.00 & 16.63 \\
\hline Hospital & 100.00 & 12.97 & Qualidade & 58.33 & 17.06 & Complexo & 80.00 & 11.19 & sozinho & 100.00 & 9.51 & Completo & 80.00 & 16.63 \\
\hline Salário & 60.00 & 12,56 & melhorar & 62.50 & 12.68 & psiquiátrico & & & Trabalhador & 36.11 & 6.64 & Estar & 80.00 & 16.63 \\
\hline Numerar & 66.67 & 9.41 & Melhor & 62.50 & 12.68 & Mental & 66.67 & 8.17 & Não & 40.91 & 4.89 & Equipe & 35.29 & 6.69 \\
\hline Reduzir & 45.45 & 5.78 & ofertar & 44.44 & 5.17 & Atender & 66.67 & 3.85 & Setor & 46.15 & 4.04 & Multi- & 66.67 & 6.02 \\
\hline mais & 34.78 & 5.67 & relacionamento & 66.67 & 5.15 & Ao & 66.67 & 3.85 & Trabalhar & 40.00 & 3.73 & profissional & & \\
\hline cuidado & 60.00 & 5.66 & Condições & 66.67 & 5.15 & Cuidar & 66.67 & 3.85 & Plantão & 50.00 & 3.11 & Familiar & 66.67 & 6.02 \\
\hline Humanizado & 60.00 & 5.66 & Capacitação & 66.67 & 5.15 & Carga & 42.86 & 2.18 & Atividade & 66.67 & 2.94 & Tranquilo & 66.67 & 6.02 \\
\hline Melhorar & 41.67 & 4.69 & Higiene & 66.67 & 5.15 & horária & & & Remanejar & 66.67 & 2.94 & Rotina & 66.67 & 6.02 \\
\hline Maior & 66.67 & 4.44 & Alimentação & 50.00 & 4.73 & Hora & 42.86 & 2.18 & Transtorno & 66.67 & 2.94 & Usuário de & 50.00 & 3.68 \\
\hline Prestar & 66.67 & 4.44 & contratar & 30.43 & 4.21 & Transtorno & 50.00 & 2.09 & mental & & & drogas & & \\
\hline Atenção & 66.67 & 4.44 & Mais & 35.71 & 4.06 & Dia & 50.00 & 2.09 & União & 66.67 & 2.94 & Chegar & 50.00 & 3.68 \\
\hline Contratar & 50.00 & 3.92 & Atendimento & 25.00 & 3.51 & Deixar & 50.00 & 2.09 & Atrasado & 66.67 & 2.94 & Quando & 25.00 & 2.48 \\
\hline Trabalhar & 30.00 & 2.11 & Trabalhar & 50.00 & 3.03 & & & & Serviço & 50.00 & 2.24 & Muito & 40.00 & 2.33 \\
\hline Plantões & 75.00 & 18.12 & Estrutura fisica & 100.00 & 30.91 & & & & Realcionamento & 44.44 & 2.16 & & & \\
\hline Material & 100.00 & 12.97 & & & & & & & & & & & & \\
\hline Não & 60.00 & 12.56 & & & & & & & & & & & & \\
\hline Atendimento & 66.67 & 9.41 & & & & & & & & & & & & \\
\hline
\end{tabular}

Figura 1. Dendrograma da Classificação Hierárquica Descendente com as partições e conteúdo do corpus da pesquisa. Fonte: dados da pesquisa. 


\section{Aspectos da sobrecarga laboral}

Com base nos termos evocados nas Classes 1, 4, e 5, que formam a categoria 1, os fatores de impacto no trabalho em um serviço psiquiátrico estavam relacionados às condições e organização do trabalho. A Classe 1 explica $21,4 \%$ do total do corpus, e teve como as palavras mais representativas: alta médica, família, complexo psiquiátrico e mental. Essa classe contempla aspectos que indicam fragilidade na relação entre a família, o paciente e a instituição. Os segmentos de textos destacados a seguir permitem identificar essa realidade, sendo, portanto, considerada, por alguns trabalhadores, como aspecto de maior sobrecarga no serviço.

Quando temos que deixar um paciente que está de alta médica em sua residência, que fica em uma outra cidade, e a família resiste em recebê-lo. É estressante, lidar com o cansaço da viagem e de ter que convencer à família a receber o paciente $(\mathrm{P} 142)$.

Quando conversamos com familiares e eles não querem aceitar a rotina do trabalho (P82).

[...] a rejeição dos familiares aos pacientes com transtornomental (P88).

Falta de apoio da família (P6).

Quanto aos fatores de maior sobrecarga, a Classe 4, que representa $26,61 \%$ do corpus analisado, constituindo a classe mais expressiva do conteúdo das respostas dos participantes, revelou que esses fatores estão atrelados às condições organizacionais e do processo de trabalho. As principais palavras desta classe foram: falta, quando, sozinho, trabalhador, não e setor. Nos fragmentos de textos descritos abaixo, é possível identificar que a falta de um membro da equipe foi considerada como evento de mais sobrecarga do trabalho no serviço investigado:

Quando um colega da equipe falta e eu tenho que trabalhar sozinha (P138).

Falta de mais pessoas do apoio e eu não gosto de ficar só com muitos pacientes para dar atenção (P177).

Trabalhar sozinho no plantão (P105).

Quando falta um trabalhador e tenho que ser remanejada para outro setor (P141).

Quando um funcionário falta e você assumi a função do outro, às vezes, até de outra profissão [...](P157).

$\mathrm{Na}$ instituição investigada, foi identificado que, dos 188 trabalhadores que participaram deste estudo, $10 \%$ declaram ter faltado pelo menos uma vez no último ano e $13 \%$ referiram ter retirado licença médica. Esses afastamentos foram resultantes de problemas organizacionais, licença maternidade e adoecimento, com registro de um caso de diagnóstico de estresse.
Neste estudo, um dos participantes afirmou que faltava este tipo de apoio no CP, associando essa falta a um aspecto do trabalho no serviço investigado que mais Ihe causava sobrecarga:

Falta de apoio psicológico e reconhecimento pela direção, se sentir abandonado (P117).

Outro fator bastante citado como grande causador de sobrecarga se refere à insuficiência de recursos humanos, materiais e/ou econômicos. Nas ilustrações expostas a seguir, é possível identificar que a sensação de sobrecarga ocorre, principalmente, pela impossibilidade de o trabalhador não poder exercer sua respectiva função ou de ofertar o atendimento ao paciente, e, portanto, considerados como um dos aspectos de mais sobrecarga no trabalho na instituição investigada:

[...] falta de materiais e recursos para desempenhar a minha função (P30).

[...] falta de lençol e material de higiene para os pacientes, dificuldade para manutenção em geral (P99).

[...]a falta de recursos (P113).

[...] a falta de material, trabalhar no improviso [...](P119).

Quando não existe material ou tem algum outro problema que impede de ter atendimento (P136).

A falta de equipamentos adequados ao atendimento do usuário [...] (P136).

Neste estudo, quando questionados aos participantes sobre os aspectos que causavam mais sobrecarga no trabalho, foram revelados como respostas os problemas de relacionamento $e$ a dificuldade do trabalho em equipe, como pode ser verificado nos fragmentos de textos a seguir:

O relacionamento com os trabalhadores, abuso de poderes [...] (P07).

Acredito que em determinadas situações a ausência de cooperação por parte do companheiro de trabalho (P63).

[...] fazer escalas. lidar com os relacionamentos entre colegas e as cobranças (P70).

Quando a equipe não fala a mesma língua (P150).

[...] falta de respeito e coleguismo da equipe multiprofissional (P160).

Quanto aos elementos de menor sobrecarga, é possível identificar tais elementos na Classe 5, que totaliza $16,1 \%$ do corpus textual. Os termos mais frequentes nesta classe foram: conversar, problema, estar, completo, equipe. Ao ser questionado sobre os aspectos que menos causavam sobrecarga no trabalho, alguns participantes consideraram que o contato com os pacientes era o aspecto que menos causavam impacto laboral. Já outros 
trabalhadores citaram os pacientes como elemento de sobrecarga, contudo, sob a condição de se fazer o uso da contenção:

Quando os pacientes não aceitam a medicação; quando há necessidade de uso de contenção e uso de medicação (P15).

O contato com os pacientes (P40).

Pacientes em crise para ser contidos (P132).

Conversar e escutar os pacientes com transtorno mental, é simplesmente maravilhoso entrar no mundo deles (P61).

Interação com os pacientes. Aprendo muito sobre as nuances da natureza humana com eles (P185).

O que menos me estressa e menos sobrecarrega é o fato de eu ver que todo meu esforço de cuidar, de dar toda assistência como técnica de enfermagem tenha valido à pena, quando vejo o paciente bem no estado geral (P55).

Embora o paciente tenha sido revelado como um dos elementos de menor sobrecarga no trabalho no CP, alguns participantes deste estudo destacaram a sobrecarga proveniente do cuidado prestado aos pacientes dependentes químicos, também denominados de usuários de drogas, conforme pode ser verificado nos fragmentos de textos dos seguintes depoimentos:

Trabalhar com dependentes químicos se torna mais difícil quando, a família é ausente e quando a equipe multiprofissional não se identifica com a área (P22).

Insistência de alguns dependentes químicos querendo atendimento. $O$ ambulatório não atende dependentes químicos (P78).

Os pacientes usuários de drogas que não estão em abstinência (P157).

Como lido com adolescentes dependentes químicos, com desvio de conduta, isso gera uma sobrecarga enorme nas resoluções dos problemas da enfermaria, sem nenhum apoio ou mudança por parte da direção (P164).

\section{Estratégias/recomendações para amenizar a sobrecarga}

Esta categoria foi originada a partir dos dados constituintes das Classes 2 e 3, representadas por 19,6\% e 17,9\% de explicação do total de corpus. Os elementos lexicais centrais foram: aumentar, hospital, salário, numerar, reduzir, mais, qualidade, melhorar, ofertar. Nesta categoria, fica explicito os aspectos do trabalho que os participantes gostariam de mudar para diminuir a sobrecarga. Pelas respostas dos trabalhadores, a sobrecarga laboral no CP só será diminuída se houver envolvimento intersetorial da rede de cuidados, melhora nas condições ambientais da instituição, maior atuação do trabalho em equipe e oferta de um trabalho digno, com direitos trabalhistas. No que diz respeito às modificações sugeridas pelos trabalhadores quanto às condições de vínculo e trabalho, destacam-se os fragmentos de textos a seguir:

Melhorar o salário e as condições de trabalho. Mais incentivo financeiro e reconhecimento (P162).

Primeiro melhorar o salário, visto ganhar menos que todo mundo, trabalhar mais e ter uma carga horária alta, para saúde mental (P164).

Em se tratando de trabalhar com portadores de transtorno mental, a carga horária e o serviço de aposentadoria deveriam ser reduzida, assim como deveríamos receber insalubridade (P156).

[...] aumentar a remuneração e ter direitos trabalhistas (P141).

Tornar-se efetivo ou com matrícula e consequentemente, melhorar a remuneração para que assim não necessitasse ter outro emprego ou fazer plantão extra (P138).

Ainda, referente à redução da sobrecarga no trabalho, foram sugeridas pelos participantes desta pesquisa, além das modificações no âmbito do CP, melhorias na articulação com outros serviços da rede de cuidados, conforme ressaltam os fragmentos a seguir:

[...] melhorar estrutura (física/materiais); melhorar a pactuação com a rede hospitalar (P76).

Serviços substitutivos funcionassem para diminuir os internamentos em hospital psiquiátricos (P06).

Os gestores do hospital e da saúde ter parceria com os municípios para conversar sobre a saúde mental em geral e dos usuários que frequentam o hospital, e sobre a responsabilidade de todos e o papel da família (P188).

No que se refere à assistência prestada no serviço, os participantes desta pesquisa consideraram que uma atuação mais efetiva do trabalhador e um melhor desempenho do trabalho em equipe contribuiria para a diminuição da sobrecarga na instituição investigada:

Um envolvimento maior com a equipe multiprofissional em defesa dos direitos dos pacientes com transtorno mental, conforme o projeto de reforma psiquiátrica (P61).

Que todos participassem ativamente nos seus setores para haver um trabalho mais organizado para nossa sobrecarga ser diminuída (P80).

[...] atendimento específicos, sem misturar pacientes usuários de drogas com pacientes portadores de transtornos mentais [...] atendimento multidisciplinar ajudaria muito no nosso trabalho (P156). 
Que cada trabalhador cumprisse os seus deveres para melhorar o trabalho e ter mais evolução. Pra não ficar só enxugando gelo [...] (P188).

É importante destacar que, conforme pode ser observado nos fragmentos de textos mencionados anteriormente, as modificações sugeridas pelos trabalhadores para diminuição da sobrecarga se associam a uma melhor oferta de cuidado ao paciente. Para elucidar essa afirmação, apresenta-se a seguir a representação gráfica, denominada de árvore (Figura 2). Essa figura demonstra os resultados da análise de similitude, possibilitando identificar as coocorrências entre as palavras e indicações da conexidade entre os termos ${ }^{16}$.

Após a análise genérica da árvore de similitude, pode-se verificar que os sentidos atribuídos à sobrecarga laboral estão relacionados ao vernáculo paciente, sendo este o elemento lexical central. Como dito anteriormente, o termo paciente, geralmente, foi citado nas respostas dos participantes, no sentido de que era preciso melhorar as condições de trabalho para ofertá-los um melhor serviço e, assim, amenizar a sobrecarga laboral. Este resultado pode ser confirmado pelos vocábulos melhorar, mais, trabalhador, quando, não, equipe, que aparecem nos seguintes fragmentos de textos:

Contratar mais funcionários e dar mais qualidade de atendimento de forma geral (P23).

Que a instituição colocasse profissionais capacitados que tivessem perfil para trabalhar na psiquiatria, pelo fato de ser um serviço diferenciado, onde os pacientes precisam
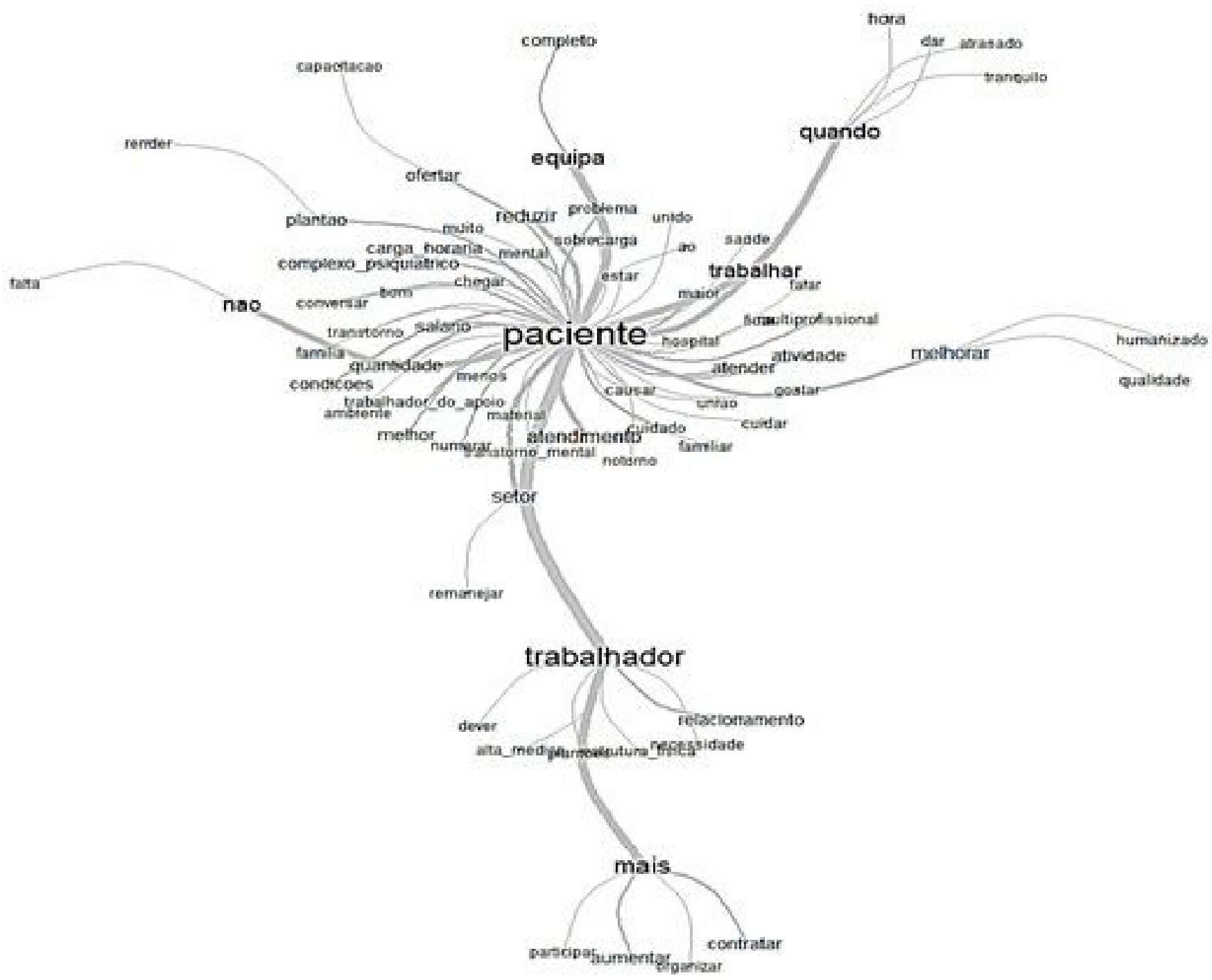

Figura 2. Análise de similitude das palavras. Fonte: dados da pesquisa. 
serem tratados com atenção, cuidado e principalmente respeito (P45).

Boas condições de trabalho para cuidar dos pacientes, pois, às vezes, falta material de higiene e roupas e fica difícil cuidar deles (P56).

Um envolvimento maior com a equipe multiprofissional em defesa dos direitos dos pacientes com transtornos mentais conforme o projeto de reforma psiquiátrica (P81).

Melhorar estrutura física e realizar uma reciclagem de profissionais, contratando pessoas que trabalham com humanização e amor (P107).

Estabelecer padrões de roupas e de melhor qualidade, melhorar a climatização, ofertar televisão para os pacientes; ter mais espaços de atividades, ofertar atividades esportivas (P150).

Redução da carga horária; atendimento específicos, sem misturar pacientes usuários de drogas com pacientes portadores de transtornos mentais [...](P156).

Que melhorasse a situação econômica do hospital para oferecer uma vida melhor aos pacientes (P163).

\section{DISCUSSÃO}

Estudos referem que a prevalência de profissionais do gênero feminino, comum nos serviços de saúde, configura, muitas vezes, em dupla jornada de trabalho, divididas entre as atividades domésticas e laborais, que induz à falta de lazer, descanso e até mesmo dedicação à família' ${ }^{12,18,19}$.

Nesta pesquisa, os trabalhadores disseram que, para suprir a ausência de algum profissional no serviço, os demais se submetiam ao excesso de atividades e, às vezes, atribuições que não eram da sua competência. Situação semelhante ocorreu em um estudo realizado com profissionais de enfermagem em serviços psiquiátricos hospitalares no Brasil, em que verificouse que a falta de profissionais ocasionou também sobrecarga laboral, recomendando a revisão do dimensionamento de pessoal, uma vez que a sobrecarga pode comprometer a qualidade do trabalho e a vida do trabalhador, o que podem ser refletidas por faltas e licenças médicas ${ }^{11,20,21}$.

O trabalho é um dos elementos mais estressores da contemporaneidade e o ambiente laboral é um dos mais propícios ao desenvolvimento de transtornos mentais ${ }^{2}$. Em um estudo realizado em um hospital psiquiátrico no nordeste do Brasil, revelou-se que o trabalho na instituição demandava um custo cognitivo crítico para adoecimento do trabalhador de enfermagem, tensão emocional e estado de alerta permanente em decorrência da natureza clínica dos pacientes. Esses foram alguns dos fatores considerados cruciais para o adoecimento ${ }^{4}$. Assim, medidas proativas destinadas a atenuar e prevenir o sofrimento psíquico e o adoecimento mental devem ser ofertadas pelas instituições aos seus trabalhadores ${ }^{6,12,22}$.
No que se refere à estrutura e recursos, a escassez de materiais exige do trabalhador o uso da criatividade e um tempo maior para elaboração de meios que auxiliem a realização do cuidado de forma eficiente. Tal condição provoca sentimentos de angústia, tensão e insatisfação no trabalhador, favorecendo o desenvolvimento de doenças, como burnout $t^{4,10,23}$.

Estudo com trabalhadores de um hospital psiquiátrico em Singapura considerou a associação entre o bem-estar psicossocial e a saúde mental positiva. Além disso, evidenciou que o local de trabalho afeta a saúde mental e o bem-estar dos profissionais ${ }^{24}$ Esse resultado corrobora o que foi revelado pelo estudo com estagiários em psiquiatria de 22 países: longas horas de trabalho, associados à baixos salários, e pouco tempo disponível para o descanso e lazer favoreceram ao desenvolvimento de burnout ${ }^{7}$.

A Síndrome de Burnout ou esgotamento profissional é um fenômeno psicossocial resultante de estressores presentes no contexto do trabalho, caracterizada pela baixa realização laboral, esgotamento emocional e físico, atitudes de indiferença e sentimento de culpa ${ }^{20}$.

Uma revisão sistemática sobre burnout em profissionais da saúde mental investigou 62 estudos em 33 países diferentes. Em todos os estudos analisados, foi constatada uma sólida relação entre o aumento da carga de trabalho e aumento das taxas de burnout, tendo como principais determinantes a carga laboral e o relacionamento entre os trabalhadores ${ }^{10}$.

Relações no trabalho caracterizadas por conflitos, imprecisão de papéis, má comunicação e problemas não resolvidos favorecem o desenvolvimento de burnout e explica muitos problemas presentes na assistência em saúde ${ }^{4,23}$. Nesse e em outros estudos, o trabalhador não se coloca no contexto do problema, apontando a responsabilidade do erro ao outro, negando-se ser parte do processo; além disso, por mais que a ocorrência do erro seja um ato individual, não pode ser separada do contexto ${ }^{25}$. Afinal, o trabalho em equipe e a oferta de cuidado dizem respeito a todos que estão em cena, e, quando todos colaboram, há diminuição de sobrecarga laboral no serviço ${ }^{15}$.

Apesar das dificuldades inerentes ao trabalho em saúde mental, existem condições percebidas pelos trabalhadores que fazem com que estes manifestem-se satisfeitos. Uma destas condições, é poder ser útil e solidário a uma população, muitas vezes, invisível para à política de saúde mental ${ }^{26}$. Sob essa ótica, ao prestar apoio aos pacientes com diagnósticos psiquiátricos, os trabalhadores encontram um sentido para si e para a vida no trabalho, percebendo o trabalho como fonte de prazer e defesa para o sofrimento ${ }^{5,9}$

Neste sentido, o contato com os pacientes pode ser considerado como fonte de prazer e o fazer contenção, repercutido em sobrecarga, como causador de sofrimento. Esse achado é compatível com o observado em uma investigação sobre a satisfação e o impacto do trabalho entre profissionais da equipe multiprofissional de um hospital psiquiátrico no Brasil. Nesta investigação os trabalhadores revelaram que se sentiam mais satisfeitos quando estavam em contato com os pacientes, 
sendo estes um dos aspectos que menos causavam impacto na instituição ${ }^{12}$.

Nesse estudo percebe-se que a sobrecarga se reflete na dificuldade dos trabalhadores em lidar com pacientes doentes em decorrência do uso de álcool, crack ou outras drogas. Supõe-se que tal dificuldade seja reflexo da falta de capacitação/ conhecimento sobre a temática, e, da frágil relação e apoio entre a família, equipe e rede de cuidado ${ }^{26}$.

Uma outra explicação é a percepção estigmatizada dos trabalhadores em relação aos usuários de drogas. Em uma pesquisa, realizada com profissionais que atuavam na assistência psiquiátrica de um hospital geral do Sul do Brasil, observou-se que: os trabalhadores consideraram os usuários de drogas culpados pela sua internação; já os pacientes com transtornos mentais de outras etiologias foram considerados vítimas, por acreditarem que eles não tinham controle sobre as suas ações. Ao se falar desses últimos, recordavam-se, primeiro, as qualidades, e só depois comentavam sobre os episódios de agressividade e delírios, necessidade de contenções físicas ou através de medicamentos ${ }^{27}$. Neste estudo, os trabalhadores apresentaram uma percepção semelhante no que diz respeito ao paciente, especialmente portador de transtorno mental, e ao uso de contenção.

É importante destacar que os trabalhadores reconhecem o risco potencial de violência provocada pelos pacientes das instituições psiquiátricas, e, nessas condições, os consideram como gerador de sobrecarga ${ }^{12}$. Neste estudo, observou-se que essa sobrecarga pode ter sido amenizada pelos trabalhadores do apoio que atuam para proteção, vigilância e segurança tanto dos usuários quanto dos próprios profissionais.

A falta de regulamentação e a perda de direitos trabalhistas e sociais, através do incentivo à legalização dos trabalhos temporários e da informalização laboral, caracterizam-se em precarização do trabalho ${ }^{28}$. Para Machado et al..$^{29}$, a precarização do trabalho produz efeitos negativos sobre a saúde mental do trabalhador, podendo se manifestar através do sofrimento social, que implica perda da confiança no outro, em si mesmo, da sua dignidade e da perspectiva de um futuro melhor. Logo, é recomendada a luta pelo trabalho digno e pela proteção à saúde e ao bem-estar.

A visão ampliada dos participantes referente aos aspectos causais e as modificações necessárias para diminuição da sobrecarga laboral vão de encontro ao que tem sido demonstrado pelos estudos sobre o trabalho nos serviços de saúde mental. A literatura aponta que, apesar do aumento do número de serviços, ainda persiste o fenômeno conhecido como porta giratória (revolving door), termo utilizado na psiquiatria para reinternações frequentes. Para combater esse fenômeno e, consequentemente, diminuir a sobrecarga nos serviços hospitalares psiquiátricos, é preciso estabelecer parcerias entre as áreas da saúde, educação, justiça, assistência social, entre outras. No espaço hospitalar psiquiátrico, deve ser trabalhado desde os aspectos ligados à macropolítica institucional até a assistência direta prestada nesse serviço ${ }^{26,30,31}$.
Embora seja relevante que, neste estudo, os participantes tenham manifestado o paciente como fator central no trabalho, é importante ressaltar que o trabalhador é um elemento-chave para o funcionamento do serviço investigado. Portanto, recomendase que sejam ofertadas abordagens eficazes para combater a sobrecarga laboral e suas consequências negativas para a qualidade de vida deste trabalhador ${ }^{12,24}$.

Um ponto relevante a ser destacado é que, neste estudo, o paciente dependente químico foi apontado como elemento de sobrecarga. Uma pesquisa realizada em hospitais psiquiátricos de Belo Horizonte, Brasil, alertou para a tendência ao aumento das internações destes pacientes nesses serviços. Sinaliza-se, portanto, a necessidade de capacitações e de adequações dos serviços, bem como da Rede de Atenção Psicossocial, para atender este público específico, amenizando o impacto no trabalho correspondente a este aspecto ${ }^{32}$.

No que se refere ao paciente, é oportuno destacar que a família é um elemento fundamental para seu cuidado, sobretudo, relevante para o processo de desospitalização, desinstitucionalização e reabilitação psicossocial ${ }^{2,19}$. Entretanto, os achados deste estudo revelaram que, quando o processo do cuidar envolve uma família ausente ou resistente ao apoio ao paciente, esta passa a ser considerada como fator de sobrecarga entre os trabalhadores.

\section{CONCLUSÕES E IMPLICAÇÕES PARA A PRÁTICA}

Como limitações para este estudo, apresentou-se a escassez em pesquisas semelhantes com a finalidade de comparar com outras realidades. Outra limitação foi, por se tratar de uma amostra convencional, as interpretações foram limitadas ao grupo de respondentes, sujeita à confiabilidade das respostas fornecidas pelos participantes da pesquisa.

Para os participantes desta pesquisa, os aspectos causadores de sobrecarga à saúde do trabalhador estavam, predominantemente, relacionados às condições laborais e organizacionais, e o paciente foi considerado como aspecto de menor sobrecarga e quase sempre, como a principal razão para as modificações elencadas no serviço para diminuição do impacto no trabalho.

Por meio deste estudo, foi possível perceber a dificuldade de o trabalhador se incluir como elemento constituinte da organização do trabalho e como sujeito que necessita de atividades de lazer e cuidados à saúde.

Assim, considerando as implicações da sobrecarga na saúde e vida do trabalhador, é fundamental que novos estudos aprofundem sobre a análise destes aspectos, especialmente sobre as estratégias referente ao bem-estar do trabalhador dentro e fora do ambiente laboral. Recomenda-se que as instituições psiquiátricas ofertem ações de incentivo à integração da equipe, valorização do trabalho e de cuidado da saúde física e mental, sobretudo, a oferta de apoio psicológico regulares, estímulo à prática de esportes e atividades sociais. 


\section{CONTRIBUIÇÕES DOS AUTORES}

Desenho do estudo. Francilene Figueirêdo da Silva Pascoal. Jaqueline Brito Vidal Batista.

Coleta ou produção dos dados. Francilene Figueirêdo da Silva Pascoal. Jaqueline Brito Vidal Batista.

Análise de dados. Francilene Figueirêdo da Silva Pascoal. Solange Fátima Geraldo da Costa. Patrícia Serpa de Souza Batista. Alana Vieira Lordão. Jaqueline Brito Vidal Batista.

Interpretação dos resultados. Francilene Figueirêdo da Silva Pascoal. Solange Fátima Geraldo da Costa. Patrícia Serpa de Souza Batista. Mariana Albernaz Pinheiro de Carvalho. Jaqueline Brito Vidal Batista.

Redação e revisão crítica do manuscrito. Francilene Figueirêdo da Silva Pascoal. Solange Fátima Geraldo da Costa. Patrícia Serpa de Souza Batista. Mariana Albernaz Pinheiro de Carvalho. Alana Vieira Lordão. Jaqueline Brito Vidal Batista.

Aprovação da versão final do artigo. Francilene Figueirêdo da Silva Pascoal. Solange Fátima Geraldo da Costa. Patrícia Serpa de Souza Batista. Mariana Albernaz Pinheiro de Carvalho. Alana Vieira Lordão. Jaqueline Brito Vidal Batista.

Responsabilidade por todos os aspectos do conteúdo e a integridade do artigo publicado. Francilene Figueirêdo da Silva Pascoal. Solange Fátima Geraldo da Costa. Patrícia Serpa de Souza Batista. Mariana Albernaz Pinheiro de Carvalho. Alana Vieira Lordão. Jaqueline Brito Vidal Batista.

\section{EDITOR ASSOCIADO}

\author{
Cristina Rosa Baixinho (1)
}

\section{EDITOR CIENTÍFICO}

\author{
Ivone Evangelista Cabral (1)
}

\section{REFERÊNCIAS}

1. Pereira AR, Joazeiro G. Percepção da internação em hospital psiquiátrico por pacientes com diagnóstico de esquizofrenia. Mundo Saude. 2015;39(4):476-83. http://dx.doi.org/10.15343/0104-7809.20153904476483.

2. Zgiet J. O trabalho alienado e a alienação mental: a reforma psiquiátrica no capitalismo. Tempus. 2018;11(3):130-43. http://dx.doi.org/10.18569/ tempus.v11i3.2433.

3. Eldal K, Veseth M, Natvik E, et al. Contradictory experiences of safety and shame in inpatient mental health practice: a qualitative study. Scand J Caring Sci. 2019;33(4):791-800. http://dx.doi.org/10.1111/scs.12674. PMid:30866094

4. Sousa KHJF, Paiva DL, Nogueira MLF, Tracera GMP, Moraes KG, Zeitoune RCG. Risco de adoecimento e custo humano no trabalho em um hospital psiquiátrico. Esc. Anna Nery Rev. Enferm. 2018;22(2):e20170288.

5. Dejours C.A psicodinâmica do trabalho na pós-modernidade. In: Mendes AM, Lima SCC, Facas EP, editores. Diálogos em psicodinâmica do trabalho. Brasília: Paralelo; 2007.

6. Yang S, Meredith P, Khan A. Stress and burnout among healthcare professionals working in a mental health setting in Singapore. Asian J Psychiatr. 2015;15:15-20. http://dx.doi.org/10.1016/j.ajp.2015.04.005. PMid:25922279.

7. Jovanović $\mathrm{N}$, Podlesek A, Volpe $U$, et al. Burnout syndrome among psychiatric trainees in 22 countries: Risk increased by long working hours, lack of supervision, and psychiatry not being first career choice. Eur Psychiatry. 2016;32:34-41. http://dx.doi.org/10.1016/j. eurpsy.2015.10.007. PMid:26802982.

8. Prins DJ, van Vendeloo S, Brand P, et al. The relationship between burnout, personality traits, and medical specialty: a national study among Dutch residents. Med Teach. 2019;41(5):584-90. http://dx.doi. org/10.1080/0142159X.2018.1514459. PMid:30394166.

9. Katsurayama M, Parente RCP, Moraes RD, Moretti-Pires RO. Work and psychic suffering in the family health strategy: a Dejouriana's perspective. Cad Saude Colet. 2013;21(4):414-9. http://dx.doi.org/10.1590/S1414462X2013000400009.

10. O'Connor K, Neff DM, Pitman S. Burnout in mental health professionals: a systematic review and meta-analysis of prevalence and determinants. Eur Psychiatry.2018;53:74-99. http://dx.doi.org/10.1016/j.eurpsy.2018.06.003. PMid:29957371.

11. Alves SR, Santos RP, Oliveira RG, Yamaguchi MU. Serviços de saúde mental: percepção da enfermagem em relação à sobrecarga e condições de trabalho. Rev Fund Care Online. 2018;10(1):25-9. http://dx.doi. org/10.9789/2175-5361.2018v10i1.25-29.

12. Dias GC, Furegato ARF. Satisfaction in, and impact of work on, the multidisciplinary team in a psychiatric hospital. Rev Enferm UERJ. 2016;24(1):e8164. http://dx.doi.org/10.12957/reuerj.2016.8164.

13. Bandeira M, Pitta AMF, Mercier C. Escalas brasileiras de avaliação da satisfação (Satis-BR) e da sobrecarga (Impacto-BR) da equipe técnica em serviços de saúde mental. J Bras Psiquiatr [Internet]. 2000; [citado 2021 fev 12];49(4):105-15. Disponível em: https://www. scielo.br/scielo.php?script=sci_nlinks\&ref=000094\&pid=S00472085200700040000700003\&lng=en

14. Camargo BV, Justo AM. IRAMUTEQ: um software gratuito para análise de dados textuais. Temas Psicol. 2013;21(2):513-8. http://dx.doi. org/10.9788/TP2013.2-16.

15. Kami MTM, Larocca LM, Chaves MMN, Lowen IMV, Souza VMP, Goto DYN. Working in the street clinic: use of IRAMUTEQ software on the support of qualitative research. Esc. Anna Nery Rev. Enferm. 2016;20(3):e20160069. http://dx.doi.org/10.5935/1414-8145.20160069.

16. Moura LKB, Marcaccini AM, Matos FTC, Sousa AFL, Nascimento GC Moura MEB. Integrative review on oral cancer. Rev. Pesqui. 2015;5:164. http://dx.doi.org/10.9789/2175-5361.2014.v6i5.164-175.

17. Bardin L. Análise de conteúdo. Lisboa: Edições 70; 2011.

18. Maciel RHMO, Santos JBF, Rodrigues RL. Condições de trabalho dos trabalhadores da saúde: um enfoque sobre os técnicos e auxiliares de nível médio. Rev Bras Saúde Ocup. 2015;40(131):75-87. http://dx.doi. org/10.1590/0303-7657000078613.

19. Barbosa VFB, Martinhago F, Hoepfner AMS, Daré PK, Caponi SNC Caponi SNC. O cuidado em saúde mental no Brasil: uma leitura a partir dos dispositivos de biopoder e biopolítica. Saúde Debate.2016;40(108):17889. http://dx.doi.org/10.1590/0103-1104-20161080015.

20. Carlotto MS, Câmara SG. Riscos psicossociais associados à síndrome de burnout em professores universitários. Avances en Psicologia Latinoamericana. 2017;35(3):447-57. http://dx.doi.org/10.12804/ revistas.urosario.edu.co/apl/a.4036.

21. Vasconcelos DV, Silva LM, Lima LAMS, et al. Absenteísmo em dois hospitais públicos de Minas Gerais: perfil epidemiológico. Rev Med Minas Gerais. 2017;27(Supl. 1):S4-10. http://dx.doi.org/10.12804/10.12804.

22. Itzhaki M, Peles-Bortz A, Kostistky H, Barnoy D, Filshtinsky V, Bluvstein I. Exposure of mental health nurses to violence associated with job stress, life satisfaction, staff resilience, and post-traumatic growth. Int J Ment Health Nurs. 2015;24(5):403-12. http://dx.doi.org/10.1111/ inm.12151. PMid:26257307.

23. Araújo MPS, Quental LLC, Medeiros SM. Working conditions: feelings of the staff and precariousness of nursing work. J Nurs UFPE On Line. 2016;10(8):2906-14. http://dx.doi.org/10.5205/1981-8963v10i8a11359p2906-2914-2016.

24. Picco L, Yuan Q, Vaingankar JA, et al. Positive mental health among health professionals working at a psychiatric hospital. PLoS One. 2017;12(6):e0178359. http://dx.doi.org/10.1371/journal.pone.0178359. PMid:28591203. 
25. Marques TP. The policy gap: global mental health in a semi-peripheral country (Portugal, 1998-2016). Interface. 2017;21(63):787-98. http:// dx.doi.org/10.1590/1807-57622016.0861.

26. Varela DSS, Sales IMM, Silva FMD, Monteiro CFS. Health network assisting users of alcohol, crack, and other drugs. Esc. Anna Nery Rev. Enferm. 2016;20(2):296-302. http://dx.doi.org/10.5935/14148145.20160040.

27. Lauermann D, Borges ZN. Saúde mental e hospitalização: qual a percepção dos profissionais da saúde? Rev Psicol UNESP [Internet] 2014; [citado 2021 fev 12];13(1):11-20. Disponível em: http://pepsic. bvsalud.org/pdf/revpsico/v13n1/a02.pdf

28. Pialarissi R. Precarização do trabalho. Rev. Adm. Saúde. 2017;17:66. http://dx.doi.org/10.23973/ras.66.11.

29. Machado FKS, Giongo CR, Mendes JMR. Terceirização e precarização do trabalho: uma questão de sofrimento social. Rev. Psicol Polit [Internet].
2016; [citado 2021 fev 12];(36):227-40. Disponível em: http://pepsic. bvsalud.org/pdf/rpp/v16n36/v16n36a07.pdf

30. Ramos DKR, Guimarães J, Enders BC. Análise contextual de reinternações frequentes de portador de transtorno mental. Interface Comum. Saúde Educ. 2011;15(37):519-27. http://dx.doi.org/10.1590/ S1414-32832011005000015.

31. Pessoa Jr JM, Santos RCA, Clementino FS, Oliveira KKD, Miranda FAN. A política de saúde mental no contexto do hospital psiquiátrico: desafios e perspectivas. Esc. Anna Nery Rev. Enferm. 2016;20(1):83-9. http://dx.doi.org/10.5935/1414-8145.20160012.

32. Coelho VAA, Volpe FM, Diniz SSL, Silva EM, Cunha CF. Alteração do perfil de atendimento dos hospitais psiquiátricos públicos de Belo Horizonte, Brasil, no contexto da reforma da assistência à saúde mental. Cien Saude Colet. 2014;19(8):3605-16. http://dx.doi.org/10.1590/141381232014198.11922013. PMid:25119099. 